\title{
Schwindel, Migräne und Schlaganfall
}

Sie kennen die Schwerpunkt-Hefte der psychoneuro und werden sich sicherlich fragen, was Schwindel, Migräne und Schlaganfall miteinander zu tun haben. Nicht viel und doch sehr viel. Es handelt sich um sehr häufige Erkrankungen die zentraler Genese sind und häufig von Nicht-Neurologen diagnostiziert und therapiert werden. Dabei erfordern gerade diese Erkrankungen ein differenziertes Vorgehen, das selbst Neurologen differenzialdiagnostische Schwierigkeiten bereitet.

In der Neurologie haben wir uns in der Vergangenheit verstärkt um seltene und differenzialdiagnostisch schwierige Fälle bemüht und etliche häufige neurologische Erkrankungen anderen Fachgruppen überlassen. Die Patienten mit Schwindel suchen häufig zuerst HNO-Ärzte und Orthopäden auf, bevor sie zu Neurologen kommen. Auch der Migräne-Patient wird genau so oft zum Orthopäden oder Augenarzt überwiesen wie zum Neurologen. Lediglich beim Schlaganfall haben es die Neurologen geschafft, dass sie in den letzten Jahren zumindest wieder die Meinungsführerschaft übernommen haben. Damit wir aber auch kompetent die Patienten versorgen, muss Fortbildung auch bei diesen Erkrankungen erfolgen. Nur weil die Erkrankung häufig ist, kann der Neurologe sie nicht zwangsläufig sicher diagnostizieren und therapieren. Dies wollen wir mit unserem Heft erreichen: Einen state-of-the-art geben, mit dem jeder überprüfen kann, ob das Wissen noch aktuell ist. Sollten sich dabei Defizite zeigen, wäre eine weiterführende Literatur zu empfehlen.

Wir haben ein sehr bewegtes Jahr hinter uns. Im nächsten Jahr werden viele Neuerungen und auch Einschnitte auf uns zukommen. Eine klare gesundheitspolitische Linie und Perspektive ist noch nicht zu erkennen. Seien wir trotzdem optimistisch und konstruktiv und versuchen wir weiter unsere Arbeit gut zu machen.

Einen guten Jahreswechsel und einen erfolgreichen Start ins neue Jahr

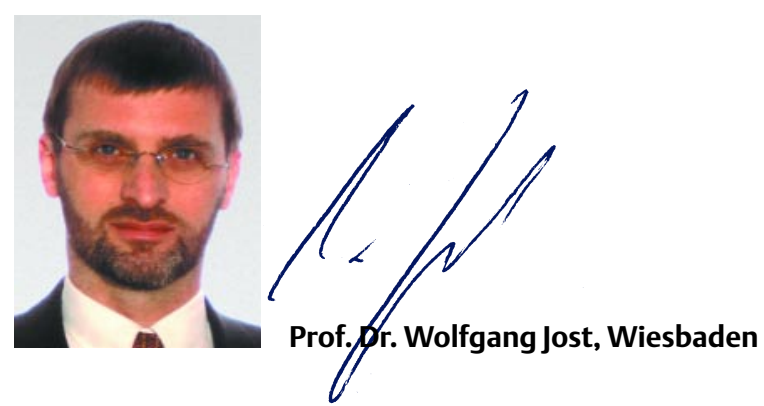

